\title{
Single edge-cracked orthotropic strip under three-point load
}

\author{
B BEHERA and A K DAS \\ Department of Mathematics, Regional Engineering College, Rourkela 769 008, India
}

\begin{abstract}
The problem of an orthotropic strip having a crack of unit length normal to one edge and subjected to a bending moment resulting from three-point loading is solved using integral transform method. The mixed boundary conditions lead to dual integral equations which are ultimately reduced to a Fredholm integral equation of second kind. The integral equation thus obtained is solved by the method developed by Fox and Goodwin. Numerical solutions for a fibre-reinforced composite material have been carried out to determine the stress intensity factor of an orthotropic medium. The same has been compared with the isotropic case.
\end{abstract}

Keywords. Edge-crack; orthotropic medium; three-point load.

\section{Introduction}

Edge-crack problems in an isotropic medium have been extensively studied due to their importance in fracture mechanics. This area of research has been enriched with the notable contributions made by Koiter (1965), Gupta \& Erdogan (1974), Bowie (1964), Kudryavstev \& Parton (1974), Ejike (1977) and others. Koiter (1965) adopted the Wiener-Hopf technique to solve his problem whereas Gupta \& Erdogan (1974) and Ejike (1973) used the Fourier transform technique. All these problems are elastically isotropic, though the edge-crack problem in anisotropic elasticity has not been widely considered except by Delate \& Erdogan (1977). In modern engineering structure designs increasing use has been made of fibre-reinforced composite materials which are elastically orthotropic. Thus, technologically, the discussion of the distribution of stresses near the crack in an orthotropic material is considered to be important and useful. In this paper an attempt has been made to determine the distribution of stresses near an edge-crack of an orthotropic strip subjected to a bending moment by a threepoint-load. This single edge-notched model is often recommended for fracture toughness testing. The isotropic counterpart of our problem has been recently studied by Ejike (1977). A general solution in terms of two potential functions has been presented.

It is assumed that the strip occupies the domain $D$ of the $x y$-plane bounded by the lines $x=0, x=c$ and the edge of the crack is defined as

and

$$
R^{+}=(x, y): y=0^{+}, 0 \leqslant x<1<c
$$

The problem of determining stresses and displacements in $D$ under a three-point load as shown in figure 1 has been solved by the method of superposition of problem I-second fundamental problem for an undamaged strip and problem II-the mixed boundary value problem for a semi-infinite strip. The mixed boundary value problem has been reduced to the solution of a Fredholm integral equation of the second kind 


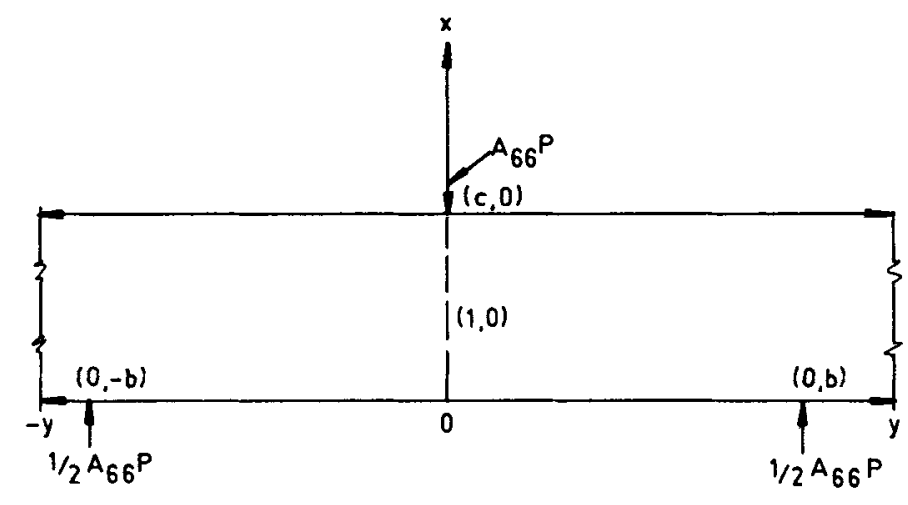

Figure 1. Edge crack under three-point load

and the quantity for the stress intensity factor has been determined. The numerical solution to the Fredholm integral equation has been obtained by the method developed by Fox \& Goodwin (1953) taking into account the material constants of boron-epoxy composite and some isotropic material. The anisotropic effect has been shown quantitatively and its influence has been discussed. As theoretically the deduction of an isotropic result from an anisotropic one is not possible, the limiting isotropic result has been calculated taking $A_{66}=0.99997$. The limiting isotropic result has also been compared with the result obtained by Ejike (1977).

\section{Basic equations}

For an orthotropic medium we choose the cartesian coordinate axes to be coincident with the principal material axes and the plain strain is defined by Satapathy $\&$ Parhi (1978)-

$$
u_{x}=u_{x}(x, y) ; \quad u_{y}=u_{y}(x, y) ; \quad u_{z}=0,
$$

where $u_{x}, u_{y}$ and $u_{z}$ are displacements in $x, y$ and $z$ directions respectively.

The stress and displacement relations in this case are as follows

$$
\begin{aligned}
& \sigma_{x x}=A_{11} \frac{\partial u_{x}}{\partial x}+A_{12} \frac{\partial u_{y}}{\partial y}, \quad \sigma_{y y}=A_{12} \frac{\partial u_{x}}{\partial x}+A_{22} \frac{\partial u_{y}}{\partial y}, \\
& \sigma_{x y}=A_{66}\left(\frac{\partial u_{x}}{\partial y}+\frac{\partial u_{y}}{\partial x}\right), \quad \sigma_{y z}=0=\sigma_{z x}
\end{aligned}
$$

where $A_{i j}$ are elastic modulii of the orthotropic material. The equations of equilibrium in terms of displacements are

$$
A_{11} \frac{\partial^{2} u_{x}}{\partial x^{2}}+A_{66} \frac{\partial^{2} u_{x}}{\partial y^{2}}+\left(A_{12}+A_{66}\right) \frac{\partial^{2} u_{y}}{\partial x \partial y}=0
$$

and

$$
A_{66} \frac{\partial^{2} u_{y}}{\partial x^{2}}+A_{22} \frac{\partial^{2} u_{y}}{\partial y^{2}}+\left(A_{12}+A_{66}\right) \frac{\partial^{2} u_{x}}{\partial x \partial y}=0
$$




\section{Displacements and stresses in terms of two potential functions}

It may be assumed that the solutions can be found in the form

$$
u_{x}=\partial \phi / \partial x, \quad u_{y}=\lambda \frac{\partial \phi}{\partial y},
$$

where $\lambda$ is a constant and $\phi$ is a function of $x$ and $y$. The equations (3) are satisfied if

$$
\frac{A_{66}+\lambda\left(A_{12}+A_{66}\right)}{A_{11}}=\frac{\lambda A_{22}}{\lambda A_{66}+A_{12}+A_{66}}=\delta^{2} \text { (say), }
$$

where $\delta^{2}$ is another constant. This yields quadratic equations, one in $\lambda$ and other in $\delta^{2}$ whose roots are $\lambda_{1}, \lambda_{2}$ and $\delta_{1}^{2}$ and $\delta_{2}^{2}$ respectively $e g \lambda_{1}$ and $\lambda_{2}$ are roots of the equation

$$
\begin{aligned}
& \lambda^{2} A_{66}\left(A_{12}+A_{66}\right)+\lambda\left[\left(A_{12}+A_{66}\right)^{2}+A_{66}^{2}-A_{11} A_{22}\right] \\
& \quad+A_{66}\left(A_{12}+A_{66}\right)=0,
\end{aligned}
$$

and $\delta_{1}^{2}, \delta_{2}^{2}$ are the roots of the equation

$$
\delta^{4} A_{11} A_{66}+\delta^{2}\left[\left(A_{12}+A_{66}\right)^{2}-A_{66}^{2}-A_{11} A_{22}\right]+A_{22} A_{66}=0 .
$$

Thus we have two potential functions $\phi_{1}$ and $\phi_{2}$ satisfying the differential equation

$$
\frac{\partial^{2} \phi_{i}}{\partial x^{2}}+\frac{\partial^{2} \phi_{i}}{\partial y_{i}^{2}}=0, \quad(i=1,2)
$$

where

$$
y_{i}=y / \delta_{i}, \quad(i=1,2)
$$

Now the displacements and stresses can be expressed in terms of two potential functions as

$$
u_{x}=\frac{\partial \phi_{1}}{\partial x}+\frac{\partial \phi_{2}}{\partial x}
$$

and

$$
\begin{aligned}
u_{y}=\frac{\lambda_{1}}{\delta_{1}} \frac{\partial \phi_{1}}{\partial y_{1}}+\frac{\lambda_{2}}{\delta_{2}} \frac{\partial \phi_{2}}{\partial y_{2}} ; \\
\frac{\sigma_{x x}}{A_{66}}=\frac{1+\lambda_{1}}{\delta_{1}^{2}} \frac{\partial^{2} \phi_{1}}{\partial x^{2}}+\frac{1+\lambda_{2}}{\delta_{2}^{2}} \frac{\partial^{2} \phi_{2}}{\partial x^{2}}, \\
\frac{\sigma_{y y}}{A_{66}}=\left(1+\lambda_{1}\right) \frac{\partial^{2} \phi_{1}}{\partial y_{1}^{2}}+\left(1+\lambda_{2}\right) \frac{\partial^{2} \phi_{2}}{\partial y_{2}^{2}}, \\
\text { and } \quad \frac{\sigma_{x y}}{A_{66}}=\frac{1+\lambda_{1}}{\delta_{1}} \frac{\partial^{2} \phi_{1}}{\partial x \partial y_{1}}+\frac{1+\lambda_{2}}{\delta_{2}} \frac{\partial^{2} \phi_{2}}{\partial x \partial y_{2}} .
\end{aligned}
$$

\subsection{Problem I}

When the orthotropic strip $0<x<c,-\infty<y<\infty$ has been subjected to three loads of magnitude $\frac{1}{2} A_{66} P, \frac{1}{2} A_{66} P$ and $A_{66} P$ at the three points $(0, b),(0,-b)$ and $(c, 0)$ respectively, let the stress and displacement components for the undamaged strip be 
denoted as $\sigma_{x x}^{0}, \sigma_{x y}^{0}, \sigma_{y y}^{0}$ and $u_{x}^{0}, u_{y}^{0}$. They can be determined from the boundary conditions

(i) $\sigma_{x y}^{0}(0, y)=0$,

(ii) $\sigma_{x x}^{0}(0, y)=-\frac{1}{2} A_{66} P\{\delta(y-b)+\delta(y+b)\}$,

(iii) $\sigma_{x y}^{0}(c, y)=0$,

and

(iv) $\sigma_{x x}^{0}(c, y)=-\frac{1}{2} A_{66} P \delta(y)$,

by the use of general Fourier transforms defined as

$$
\bar{\phi}(x, \zeta)=(2 \pi)^{-1 / 2} \int_{-\infty}^{\infty} \phi(x, y) \exp (i \zeta y) \mathrm{d} y .
$$

Here in (12) $\delta(y)$ denotes the Dirac delta distribution.

The transformed boundary conditions (12) are

(i) $\bar{\sigma}_{x y}^{0}(0, \xi)=0$,

(ii) $\bar{\sigma}_{x x}^{0}(0, \xi)=-(2 \pi)^{-1 / 2} A_{66} P \cos (b \xi)$,

(iii) $\bar{\sigma}_{x y}^{0}(c, \xi)=0$,

(iv) $\bar{\sigma}_{x x}^{0}(c, \xi)=-(2 \pi)^{-1 / 2} A_{66} P$.

To solve this problem we choose two potential functions $\phi_{j}(x, y),(j=1,2)$ to be

$$
\begin{aligned}
& \phi_{j}(x, y)=(2 \pi)^{-1 / 2} \int_{-\infty}^{\infty}|\xi|^{-2}\left[A_{j}(\xi) \exp \left(-x|\xi| \delta_{j}\right)\right. \\
& \left.+B_{j}(\xi) \exp \left\{-(c-x)|\xi| \delta_{j}\right\}\right] \exp (-i \xi y) \mathrm{d} \xi, \quad(j=1,2)
\end{aligned}
$$

where $A_{j}(\xi), B_{j}(\xi), j=(1,2)$ are unknown functions of $\xi$ to be determined from the boundary conditions (13)

Utilising the boundary conditions (i) to (iv) of (13) we determine $A_{1}(\xi), B_{1}(\xi), A_{2}(\xi)$ and $B_{2}(\xi)$ as

$$
\begin{aligned}
A_{1}(\xi)= & \frac{(2 \pi)^{-1 / 2} P \delta_{2}}{1+\lambda_{1}}\left[\cos (b \xi)\left\{X-Y \exp \left(-c|\xi| \delta_{2}\right)\right\}\right. \\
& \left.+Y-X \exp \left(-c|\xi| \delta_{2}\right)\right] \Delta(c \xi) \\
B_{1}(\xi)= & \frac{(2 \pi)^{-1 / 2} P \delta_{2}}{1+\lambda_{1}}\left[\cos (b \xi)\left\{Y-\exp \left(-c|\xi| \delta_{2}\right)\right\}\right. \\
& \left.+X-Y \exp \left(-c|\xi| \delta_{2}\right)\right] \Delta(c \xi) \\
A_{2}(\xi)= & \frac{(2 \pi)^{-1 / 2} P \delta_{1}}{1+\lambda_{2}}\left[\cos (b \xi)\left\{Y \exp \left(-c|\xi| \delta_{1}\right)-X\right\}\right. \\
& \left.+X \exp \left(-c|\xi| \delta_{1}\right)-Y\right] \Delta(c \xi)
\end{aligned}
$$

and

$$
\begin{aligned}
B_{2}(\xi)= & \frac{(2 \pi)^{-1 / 2} P \delta_{1}}{1+\lambda_{2}}\left[\cos (b \xi)\left\{X \exp \left(-c|\xi| \delta_{1}\right)-Y\right\}\right. \\
& \left.+Y \exp \left(-c|\xi| \delta_{1}\right)-X\right] \Delta(c \xi)
\end{aligned}
$$


where $X$ and $Y$ are known functions of $\xi$ written as

$$
\begin{aligned}
X= & \left(\delta_{1}-\delta_{2}\right)\left[1-\exp \left\{-c \xi\left(\delta_{1}+\delta_{2}\right)\right\}\right], \\
Y= & \left(\delta_{1}+\delta_{2}\right)\left[\exp \left(-c|\xi| \delta_{1}\right)-\exp \left(-c|\xi| \delta_{2}\right)\right], \text { and } \\
& \Delta(c \xi)=\left[X^{2}-Y^{2}\right]^{-1} .
\end{aligned}
$$

Hence the components of stress and displacement are obtained from (11) and (10) using (15) as

$$
\begin{aligned}
& \frac{\sigma_{x x}^{0}(x, y)}{A_{66}}=\frac{P \delta_{2}}{\pi} \int_{0}^{\infty}\left[\left\{\cos (b \xi)\left(X-Y \exp \left(-c \xi \delta_{2}\right)\right)\right.\right. \\
& \left.+Y-X \exp \left(-c \xi \delta_{1}\right)\right\} \exp \left(-x \xi \delta_{1}\right)+\{\cos (b \xi) \\
& \left.\times\left(Y-X \exp \left(-c \xi \delta_{2}\right)\right)+X-Y \exp \left(-c \xi \delta_{2}\right)\right\} \\
& \times \exp \left(-(c-x) \xi \delta_{1}\right)+\left\{\cos (b \xi)\left(Y \exp \left(-c \xi \delta_{1}\right)-X\right)\right. \\
& \left.+X \exp \left(-c \xi \delta_{1}\right)-Y\right\} \exp \left(-x \xi \delta_{2}\right) \\
& +\left\{\cos (b \xi)\left(X \exp \left(-c \xi \delta_{1}\right)-Y\right)+Y \exp \left(-c \xi \delta_{1}\right)-X\right\} \\
& \left.\exp \left(-(c-x) \xi \delta_{2}\right)\right] \Delta(c \xi) \cos (\xi y) \mathrm{d} \xi \text {. } \\
& \frac{\sigma_{x y}^{0}(x, y)}{A_{66}}=\frac{P \delta_{2}}{\pi} \int_{0}^{\infty}\left\langle\delta _ { 1 } \left[\left\{\cos (b \xi)\left(X-Y \exp \left(-c \xi \delta_{2}\right)\right)+Y-X\right.\right.\right. \\
& \left.\times \exp \left(-c \xi \delta_{2}\right)\right\} \exp \left(-x \xi \delta_{1}\right)-\left\{\cos (b \xi)\left(Y-X \exp \left(-c \xi \delta_{2}\right)\right)\right. \\
& \left.\left.+X-Y \exp \left(-c \xi \delta_{2}\right)\right\} \exp \left(-(c-x) \xi \delta_{1}\right)\right]+\delta_{2}[\{\cos (b \xi) \\
& \left.\times\left(Y \exp \left(-c \xi \delta_{1}\right)-X\right)+X \exp \left(-c \xi \delta_{1}\right)-Y\right\} \exp \left(-x \xi \delta_{2}\right) \\
& -\left\{\cos (b \xi)\left(Y-X \exp \left(-c \xi \delta_{2}\right)\right)+X-Y \exp \left(-c \xi \delta_{2}\right)\right\} \\
& \left.\times \exp \left(-(c-x) \xi \delta_{2}\right)\right]>\Delta(c \xi) \sin (\xi y) \mathrm{d} \xi \text {. } \\
& \frac{\sigma_{y y}^{0}(x, y)}{A_{66}}=\frac{-P \delta_{1} \delta_{2}}{\pi} \int_{0}^{\infty}\left\langle\delta _ { 1 } \left[\left\{\cos (b \xi)\left(X-Y \exp \left(-c \xi \delta_{2}\right)\right)\right.\right.\right. \\
& \left.+Y-X \exp \left(-c \xi \delta_{2}\right)\right\} \exp \left(-x \xi \delta_{1}\right)+\{\cos (b \xi) \\
& \left.\times\left(Y-X \exp \left(-c \xi \delta_{2}\right)\right)+X-Y \exp \left(-c \xi \delta_{2}\right)\right\} \\
& \left.x \exp \left(-(c-x) \xi \delta_{1}\right)\right]+\delta_{2}\left[\left\{\cos (b \xi)\left(Y \exp \left(-c \xi \delta_{1}\right)-X\right)\right.\right. \\
& \left.+X \exp \left(-c \xi \delta_{1}\right)-Y\right\} \exp \left(-x \xi \delta_{2}\right) \\
& +\left\{\cos (b \xi)\left(X \exp \left(-c \xi \delta_{1}\right)-Y\right)+Y \exp \left(-c \xi \delta_{1}\right)-X\right\} \\
& \left.x \exp \left(-(c-x) \xi \delta_{2}\right)\right]>\Delta(c \xi) \cos (\xi y) \mathrm{d} \xi \text {. } \\
& u_{x}^{0}(x, y)=\frac{-P}{\pi\left(1+\lambda_{1}\right)\left(1+\lambda_{2}\right)} \int_{0}^{\infty} \xi^{-1}\left\langle\delta_{1}\left(1+\lambda_{2}\right)[\{\cos (b \xi)\right. \\
& \left.\times\left(\mathrm{X}-Y \exp \left(-c \xi \delta_{2}\right)\right)+Y-X \exp \left(-c \xi \delta_{2}\right)\right\} \exp \left(-x \xi \delta_{1}\right) \\
& -\left\{\cos (b \xi)\left(Y-X \exp \left(-c \xi \delta_{2}\right)\right)+X-Y \exp \left(-c \xi \delta_{2}\right)\right\} \\
& \left.x \exp \left(-(c-x) \xi \delta_{1}\right)\right]+\delta_{1}\left(1+\lambda_{1}\right)[\{\cos (b \xi)
\end{aligned}
$$




$$
\begin{aligned}
& \left.\times\left(Y \exp \left(-c \xi \delta_{1}\right)-X\right)+X \exp \left(-c \xi \delta_{1}\right)-Y\right\} \exp \left(-x \xi \delta_{2}\right) \\
& -\left\{\cos (b \xi)\left(X \exp \left(-c \xi \delta_{1}\right)-Y\right)+Y \exp \left(-c \xi \delta_{1}\right)-X\right\} \\
& \left.\times \exp \left(-(c-x) \xi \delta_{2}\right)\right]>\Delta(c \xi) \cos (\xi y) \mathrm{d} \xi \\
u_{y}^{0}(x, y)= & \frac{-P}{\pi\left(1+\lambda_{1}\right)\left(1+\lambda_{2}\right)} \int_{0}^{\infty} \xi^{-1}\left\langle\delta_{2}\left(1+\lambda_{2}\right) \lambda_{1}[\{\cos (b \xi)\right. \\
& \left.\times\left(X-Y \exp \left(-c \xi \delta_{2}\right)\right)+Y-X \exp \left(-c \xi \delta_{2}\right)\right\} \exp \left(-x \xi \delta_{1}\right) \\
& +\left\{\cos (b \xi)\left(Y-X \exp \left(-c \xi \delta_{2}\right)\right)+X-Y \exp \left(-c \xi \delta_{2}\right)\right\} \\
& \left.\times \exp \left(-(c-x) \xi \delta_{1}\right)\right]+\lambda_{2}\left(1+\lambda_{1}\right) \delta_{1}[\{\cos (b \xi) \\
& \left.\left(Y \exp \left(-c \xi \delta_{1}\right)-X\right)+X \exp \left(-c \xi \delta_{1}\right)-Y\right\} \exp \left(-x \xi \delta_{2}\right) \\
& +\left\{\cos (b \xi)\left(X \exp \left(-c \xi \delta_{1}\right)-Y\right)+Y \exp \left(-c \xi \delta_{1}\right)-X\right\} \\
& \left.\times \exp \left(-(c-x) \xi \delta_{2}\right)\right]>\Delta(c \xi) \sin (\xi y) \mathrm{d} \xi
\end{aligned}
$$

It follows from (17), (20) and (18) that

$$
\begin{aligned}
&\left.\begin{array}{c}
\sigma_{x y}^{0}(x, 0)= \\
u_{y}^{0}(x, 0)=
\end{array}\right\} \quad 0 \leqslant x<c \\
& \sigma_{y y}^{0}(x, 0)= \frac{-P \delta_{1} \delta_{2} A_{66}}{\pi} \int_{0}^{\infty}\left\langle\delta _ { 1 } \left[\left\{\cos (b \xi)\left(X-Y \exp \left(-c \xi \delta_{2}\right)\right)\right.\right.\right. \\
&\left.+Y-X \exp \left(-c \xi \delta_{2}\right)\right\} \exp \left(-x \xi \delta_{1}\right) \\
&+\left\{\cos (b \xi)\left(Y-X \exp \left(-c \xi \delta_{2}\right)\right)\right. \\
&\left.\left.+X-Y \exp \left(-c \xi \delta_{2}\right)\right\} \exp \left(-(c-x) \xi \delta_{1}\right)\right] \\
&+\delta_{2}\left[\left\{\cos (b \xi)\left(Y \exp \left(-c \xi \delta_{1}\right)-X\right)+X \exp \left(-c \xi \delta_{1}\right)-Y\right\}\right. \\
& \times \exp \left(-x \xi \delta_{2}\right)+\left\{\operatorname { c o s } ( b \xi ) \left(X \exp \left(-c \xi \delta_{1}\right)-Y\right.\right. \\
&\left.+Y \exp \left(-c \xi \delta_{1}\right)-X\right\} \exp \left(-(c-x) \xi \delta_{2}\right]>\Delta(c \xi) \mathrm{d} \xi
\end{aligned}
$$

\subsection{Problem II}

The complementary problem of problem $I$ is the present mixed boundary value problem of determining the stresses and displacements for the damaged strip with its edges stress-free and the faces of the crack acted upon by tractions that occur in the undamaged strip. The components of stress and displacements for semi-infinite strip $0<x<c, y>0$ are denoted as $\sigma_{x x}^{1}, \sigma_{x y}^{1}, \sigma_{y y}^{1}$ and $u_{x}^{1}, u_{y}^{1}$ which can be determined from the boundary conditions prescribed as

(i) $\sigma_{x x}^{1}(0, y)=0$

(ii) $\sigma_{x x}^{1}(c, y)=0$

(iii) $\sigma_{x y}^{1}(0, y)=0$

$0<y<\infty$

(iv) $\sigma_{x y}^{1}(c, y)=0$

(v) $\sigma_{x y}^{1}(x, 0)+\sigma_{x y}^{0}(x, 0)=0,0<x<c$, 


$$
\begin{aligned}
& \text { (vi) } \sigma_{y y}^{1}(x, 0)+\sigma_{y y}^{0}(x, 0)=0, \quad 0<x<1, \\
& \text { (vii) } u_{y}^{1}(x, 0)+u_{y}^{0}(x, 0)=0, \quad 1 \leqslant x \leqslant c
\end{aligned}
$$

Let the Fourier cosine and sine transforms be defined as

$$
\mathscr{F}_{c} f(\xi) ; \xi \rightarrow x=(2 / \pi)^{1 / 2} \int_{0}^{\infty} f(\xi) \cos (\xi x) \mathrm{d} \xi,
$$

and

$$
\mathscr{F}_{s} f(\xi) ; \xi \rightarrow x=(2 / \pi)^{1 / 2} \int_{0}^{\infty} f(\xi) \sin (\xi x) \mathrm{d} \xi .
$$

For the solution of the mixed boundary value problem we choose two suitable potential functions $\phi_{i}(x, y),(i=1,2)$ as

$$
\begin{gathered}
\phi_{i}(x, y)=\mathscr{F}_{\mathrm{c}}\left[\zeta^{-2} C_{i}(\zeta) \exp \left(-y_{i} \zeta\right) ; \zeta \rightarrow x\right]+\mathscr{F}_{\mathrm{c}}\left[\xi ^ { - 2 } \left\{D_{i}(\xi)\right.\right. \\
\left.\left.\operatorname{ch}\left(\xi \delta_{i} x\right)+E_{i}(\xi) \exp \left(-x \xi \delta_{i}\right)\right\} ; \xi \rightarrow y\right],
\end{gathered}
$$

where the unknown functions $C_{i}(\zeta), D_{i}(\xi)$ and $E_{i}(\xi)$ for $(i=1,2)$ are to be determined from the above boundary conditions.

The stress and displacement components can be determined from (11) and (10) using (24).

From the boundary condition (v) of (23) we obtain

$$
C_{1}(\zeta)=-\frac{\left(1+\lambda_{2}\right) \delta_{1}}{\left(1+\lambda_{1}\right) \delta_{2}} C_{2}(\zeta)
$$

Also from condition (iii) of (23) we have

$$
E_{1}(\zeta)=-\frac{\left(1+\lambda_{2}\right) \delta_{2}}{\left(1+\lambda_{1}\right) \delta_{1}} E_{2}(\zeta)
$$

Now with the help of (25) and (26) we can get the final form of components of stress and displacement as:

$$
\begin{aligned}
\frac{\sigma_{x x}^{1}(x, y)}{A_{66}}= & \frac{1+\lambda_{2}}{\delta_{1} \delta_{2}^{2}} \mathscr{F}_{c}\left[C_{2}(\zeta)\left\{\delta_{2} \exp \left(-y_{1} \zeta\right)-\delta_{1} \exp \left(-y_{2} \zeta\right)\right\} ;\right. \\
& \zeta \rightarrow x]+\mathscr{F}_{c}\left[\left\{\left(1+\lambda_{1}\right) D_{1}(\xi) \operatorname{ch}\left(\xi \delta_{1} x\right)+\left(1+\lambda_{2}\right) D_{2}(\xi)\right\}\right. \\
& \times \operatorname{ch}\left(\xi \delta_{2} x\right)+\frac{1+\lambda_{2}}{\delta_{1}} E_{2}(\xi)\left(\delta_{1} \exp \left(-x \xi \delta_{2}\right)\right. \\
\text { and } \quad & \left.\left.-\delta_{2} \exp \left(-x \xi \delta_{1}\right)\right) ; \xi \rightarrow y\right] ; \\
\frac{\sigma_{x y}^{1}(x, y)}{A_{66}}= & \frac{1+\lambda_{2}}{\delta_{1}}\left[\mathscr{F}_{s} C_{2}(\zeta)\left\{\exp \left(-y_{2} \zeta\right)-\exp \left(-y_{1} \zeta\right)\right\} ; \zeta \rightarrow x\right] \\
& -\mathscr{F}_{s}\left[\left(1+\lambda_{1}\right) \delta_{1} D_{1}(\xi) \operatorname{sh}\left(\xi \delta_{1} x\right)+\left(1+\lambda_{2}\right) \delta_{2} D_{2}(\xi) \operatorname{sh}\left(\xi \delta_{2} x\right)\right. \\
& \left.-\left(1+\lambda_{2}\right) \delta_{2} E_{2}(\xi)\left\{\exp \left(-x \xi \delta_{2}\right)-\exp \left(-x \xi \delta_{1}\right)\right\} ; \xi \rightarrow \mathrm{y}\right], \quad \\
\frac{\sigma_{y y}^{1}(x, y)}{A_{66}}= & \frac{1+\lambda_{2}}{\delta_{2}} \mathscr{F}_{c}\left[C_{2}(\zeta)\left\{\delta_{2} \exp \left(-y_{2} \zeta\right)-\delta_{1} \exp \left(-y_{1} \zeta\right) ; \zeta \rightarrow x\right]\right.
\end{aligned}
$$




$$
\begin{aligned}
& -F_{c}\left[\left\{\left(1+\lambda_{1}\right) \delta_{1}^{2} D_{1}(\xi) \operatorname{ch}\left(\xi \delta_{1} x\right)+\left(1+\lambda_{2}\right) \delta_{2}^{2} D_{2}(\xi) \operatorname{ch}\left(\xi \delta_{2} x\right)\right.\right. \\
& +\left(1+\lambda_{2}\right) \delta_{2} E_{2}(\xi) \delta_{2} \exp \left(-x \xi \delta_{2}\right) \\
& \left.\left.-\delta_{1} \exp \left(-x \xi \delta_{1}\right)\right\} ; \xi \rightarrow y\right] \\
u_{x}^{1}(x, y)= & \frac{-1}{\left(1+\lambda_{2}\right) \delta_{2}}\left[\zeta ^ { - 1 } C _ { 2 } ( \zeta ) \left\{\left(1+\lambda_{1}\right) \delta_{2} \exp \left(-y_{2} \zeta\right)-\left(1+\lambda_{2}\right) \delta_{1}\right.\right. \\
& \left.\exp \left(-y_{1} \zeta\right)\right\} \\
& \zeta \rightarrow x+F_{c}\left\langle\xi^{-1} \delta_{1} D_{1}(\xi) \operatorname{sh}\left(\xi \delta_{1} x\right)+\delta_{2} D_{2}(\xi) \operatorname{sh}\left(\xi \delta_{2} x\right)\right. \\
& +\frac{\delta_{2}}{1+\lambda_{1}} E_{2}(\xi)\left\{\left(1+\lambda_{1}\right) \exp \left(-x \xi \delta_{2}\right)\right. \\
& \left.\left.\left.-\left(1+\lambda_{2}\right) \exp \left(-x \xi \delta_{1}\right)\right\}\right] ; \xi \rightarrow y\right\rangle \\
& -1 \\
u_{y}^{1}(x, y)= & \left(1+\lambda_{1}\right) \delta_{2} \\
& \left.\left.-\lambda_{1}\left(1+\lambda_{2}\right) \exp \left(-\zeta_{1} \zeta\right)\right\} ; \zeta \rightarrow x\right]-F_{s}\left\langle\xi ^ { - 1 } \left[\lambda_{1} D_{1}(\xi) \operatorname{ch}\left(\xi \delta_{1} x\right)\right.\right. \\
& +\lambda_{2} D_{2}(\xi) \operatorname{ch}\left(\xi \delta_{2} x\right)+\frac{E_{2}(\xi)}{\left(1+\lambda_{1}\right) \delta_{1}}\left(1+\lambda_{1}\right) \delta_{1} \lambda_{2} \exp \left(-x \xi \delta_{2}\right) \\
& \left.\left.\left.-\left(1+\lambda_{2}\right) \delta_{2} \lambda_{1} \exp \left(-x \xi \delta_{2}\right)\right\}\right] ; \xi \rightarrow y\right\rangle
\end{aligned}
$$

Now the boundary condition (vi) of (23) gives

$$
\begin{aligned}
& \frac{\left(\delta_{1}-\delta_{2}\right)\left(1+\lambda_{2}\right)}{\delta_{2}} \mathscr{F}_{c}\left[C_{2}(\zeta) ; \zeta \rightarrow x\right]-(2 / \pi)^{1 / 2} \int_{0}^{\infty}\left[\left(1+\lambda_{1}\right) \delta_{1}^{2} D_{1}(\xi)\right. \\
& \operatorname{ch}\left(\xi \delta_{1} x\right)+\left(1+\lambda_{2}\right) \delta_{2}^{2} D_{2}(\xi) \operatorname{ch}\left(\xi \delta_{2} x\right)+\left(1+\lambda_{2}\right) \delta_{2} E_{2}(\xi) \\
& \left.\left\{\delta_{2} \exp \left(-x \xi \delta_{2}\right)-\delta_{1} \exp \left(-x \xi \delta_{1}\right)\right\}\right] \mathrm{d} \xi \\
& =\frac{-1}{A_{66}} \sigma^{0}(b, c ; x) \text { for } 0<x<1 .
\end{aligned}
$$

Integrating both the sides of (32) with respect to $x$ from limit 0 to $x$ we obtain

$$
\begin{aligned}
& \frac{\left(\delta_{1}-\delta_{2}\right)\left(1+\lambda_{2}\right)}{\delta_{2}} \mathscr{F}_{s}\left[\zeta^{-1} C_{2}(\zeta) ; \zeta \rightarrow x\right]=-(2 / \pi)^{1 / 2} \int_{0}^{\infty} \xi^{-1} \\
& \quad\left[\left(1+\lambda_{1}\right) \delta_{1} D_{1}(\xi) \operatorname{sh}\left(\xi \delta_{1} x\right)+\left(1+\lambda_{2}\right) \delta_{2} D_{2}(\xi) \operatorname{sh}\left(\xi \delta_{2} x\right)\right. \\
& \left.+\left(1+\lambda_{2}\right) \delta_{2} E_{2}(\xi)\left\{\exp \left(-x \xi \delta_{1}\right)-\exp \left(-x \xi \delta_{2}\right)\right\}\right] \mathrm{d} \xi \\
& +\frac{1}{A_{66}} \int_{0}^{x} \sigma^{0}(b, c, v) \mathrm{d} v \text { for } 0<x<1 .
\end{aligned}
$$

Also from condition (vii) of (23) we obtain

$$
\frac{\lambda_{1}-\lambda_{2}}{\left(1+\lambda_{1}\right) \delta_{2}} \mathscr{F}_{c}\left[\zeta^{-1} C_{2}(\zeta) ; \zeta \rightarrow x\right]=0
$$

In order to solve the integral equations (33) and (34) we take the solution

$$
C_{2}(\zeta)=\zeta \int_{0}^{1} \phi(t) J_{0}(\zeta t) \mathrm{d} t, \phi(0)=0 .
$$


Equation (34) is satisfied identically while (33) after a little manipulation is reduced to Fredholm integral equation

$$
\begin{aligned}
\phi(t)= & \frac{-\delta_{2} t}{\left(1+\lambda_{2}\right)\left(\delta_{1}-\delta_{2}\right)} \int_{0}^{\infty}\left[\left(1+\lambda_{1}\right) \delta_{1}^{2} D_{1}(\xi) I_{0}\left(t \xi \delta_{1}\right)+\left(1+\lambda_{2}\right) \delta_{2}^{2}\right. \\
& \times D_{2}(\xi) I_{0}\left(t \xi \delta_{2}\right)+\left(1+\lambda_{2}\right) \delta_{2} E_{2}(\xi)\left\{\delta_{2} M_{0}\left(t \xi \delta_{2}\right)\right. \\
& \left.\left.-\delta_{1} M_{0}\left(t \xi \delta_{1}\right)\right\}\right] \mathrm{d} \xi \\
& +(2 / \pi)^{1 / 2} \frac{\delta_{2}}{A_{66}\left(1+\lambda_{2}\right)\left(\delta_{1}-\delta_{2}\right)} \frac{\mathrm{d}}{\mathrm{d} t} \int_{0}^{t} \frac{x \mathrm{~d} x}{\left(t^{2}-x^{2}\right)^{1 / 2}} \\
& \times \int_{0}^{x} \sigma^{0}(b, c ; v) \mathrm{d} v
\end{aligned}
$$

with the help of the following formulae

$$
\begin{aligned}
& \frac{2}{\pi} \frac{\mathrm{d}}{\mathrm{d} t} \int_{0}^{t} \frac{x \operatorname{sh}\left(\xi x \delta_{i}\right) \mathrm{d} x}{\left(t^{2}-x^{2}\right)^{1 / 2}}=t \xi \delta_{i} I_{0}\left(t \xi \delta_{i}\right) \\
& \frac{2}{\pi} \frac{\mathrm{d}}{\mathrm{d} t} \int_{0}^{t} \frac{x \exp \left(-x \xi \delta_{i}\right) \mathrm{d} x}{\left(t^{2}-x^{2}\right)^{1 / 2}}=\frac{2}{\pi}-t \xi \delta_{i} M_{0}\left(t \xi \delta_{i}\right)
\end{aligned}
$$

where $\quad M_{0}(z)=I_{0}(z)-L_{0}(z)$,

and $I_{0}(z)$ and $L_{0}(z)$ are the modified Bessel's and modified Struve's functions respectively. Now from the boundary conditions (i), (ii) and (iv) of (23) we get the following equations

$$
\begin{aligned}
& \delta_{1}\left(1+\lambda_{1}\right) D_{1}(\xi)+\delta_{1}\left(1+\lambda_{2}\right) D_{2}(\xi)+\left(\delta_{1}-\delta_{2}\right)\left(1+\lambda_{2}\right) E_{2}(\xi)=F_{0}(\xi), \\
& \delta_{1}\left(1+\lambda_{1}\right) D_{1}(\xi) \operatorname{ch}\left(\xi \delta_{1} c\right)+\delta_{1}\left(1+\lambda_{2}\right) \operatorname{ch}\left(\xi \delta_{2} c\right) D_{2}(\xi)+\left(1+\lambda_{2}\right) \\
& \quad\left\{\delta_{1} \exp \left(-c \xi \delta_{2}\right)-\delta_{2} \exp \left(-c \xi \delta_{1}\right)\right\} E_{2}(\xi)=F_{1}(\xi)
\end{aligned}
$$

and

$$
\begin{gathered}
\delta_{1}\left(1+\lambda_{1}\right) \operatorname{sh}\left(\xi \delta_{1} c\right) D_{1}(\xi)+\delta_{2}\left(1+\lambda_{2}\right) \operatorname{sh}\left(\xi \delta_{2} c\right) D_{2}(\xi)+\left(1+\lambda_{2}\right) \delta_{2} \\
\quad\left\{\exp \left(-c \xi \delta_{1}\right)-\exp \left(-c \xi \delta_{2}\right)\right\} E_{2}(\xi)=F_{2}(\xi),
\end{gathered}
$$

where

$$
\begin{aligned}
F_{0}(\xi)= & -\frac{\delta_{1}}{\delta_{2}}\left(1+\lambda_{2}\right) \xi \int_{0}^{1}\left[\delta_{2} M_{0}\left(r \xi \delta_{2}\right)-\delta_{1} M_{0}\left(r \xi \delta_{1}\right)\right] \phi(r) \mathrm{d} r \\
F_{1}(\xi)= & -\frac{\delta_{1}}{\delta_{2}}\left(1+\lambda_{2}\right) \xi \int_{0}^{1}\left[\delta_{2} \exp \left(-c \xi \delta_{2}\right) I_{0}\left(r \xi \delta_{2}\right)\right. \\
& \left.-\delta_{1} \exp \left(-c \xi \delta_{1}\right) I_{0}\left(r \xi \delta_{1}\right)\right] \phi(r) \mathrm{d} r
\end{aligned}
$$

and $\quad F_{2}(\xi)=\frac{1+\lambda_{2}}{\delta_{2}} \xi \int_{0}^{1}\left[\delta_{2}^{2} \exp \left(-c \xi \delta_{2}\right) I_{0}\left(r \xi \delta_{2}\right)\right.$

$$
\left.-\delta_{1}^{2} \exp \left(-c \xi \delta_{1}\right) I_{0}\left(r \xi \delta_{1}\right)\right] \phi(r) \mathrm{d} r .
$$

Solving (38), (39) and (40) for $D_{1}(\xi), D_{2}(\xi)$ and $E_{2}(\xi)$ we obtain

$$
D_{1}(\xi)=-2 \frac{1+\lambda_{2}}{1+\lambda_{1}} \frac{\xi}{p_{0}(\xi)}\left[\delta_{2}\left\{n \exp (m c \xi)-m \exp (-n c \xi)-2 \delta_{1}\right)\right\}
$$




$$
\begin{aligned}
& \int_{0}^{1}\left\{\delta_{2} M_{0}\left(r \xi \delta_{2}\right)-\delta_{1} M_{0}\left(r \xi \delta_{1}\right)\right\} \phi(r) \mathrm{d} r+2 \delta_{2}^{2} m \exp (-n c \xi) \\
& \int_{0}^{1} I_{0}\left(r \xi \delta_{2}\right) \phi(r) \mathrm{d} r-m n\{\exp (-n c \xi)+\exp (m c \xi)\} \\
& \left.\times \int_{0}^{1} I_{0}\left(r \xi \delta_{1}\right) \phi(r) \mathrm{d} r\right] \\
D_{2}(\xi)= & -2 \frac{\delta_{1} \xi}{\delta_{2} p_{0}(\xi)}\left[\left\{n \exp (-m c \xi)+m \exp (-n c \xi)-2 \delta_{2}\right\}\right. \\
& \times \int_{0}^{1}\left\{\delta_{2} M_{0}\left(r \xi \delta_{2}\right)-\delta_{1} M_{0}\left(r \xi \delta_{1}\right)\right\} \phi(r) \mathrm{d} r-\frac{\delta_{1}}{\delta_{2}} m n \\
& \times\{\exp (-n c \xi)-\exp (-m c \xi)\} \int_{0}^{1} I_{0}\left(r \xi \delta_{2}\right) \phi(r) \mathrm{d} r \\
& \left.-2 \delta_{1}\{m+n \exp (-n c \xi)\} \int_{0}^{1} I_{0}\left(r \xi \delta_{1}\right) \phi(r) \mathrm{d} r\right] \\
\text { and } \quad & \frac{\delta_{1}}{\delta_{2} p_{0}(\xi)}[\{m(\exp (n c \xi)-\exp (-n c \xi))-n \exp (-m c \xi) \\
& -\exp (m c \xi)\} \int_{0}^{1}\left\{\delta_{2} M_{0}\left(r \xi \delta_{2}\right)-\delta_{1} M_{0}\left(r \xi \delta_{1}\right)\right\} \phi(r) \mathrm{d} r+\delta_{2} \\
& \left.+\delta_{1}\left\{2 \delta_{1}-n \exp (m c \xi)+m \exp (-n c \xi)\right\} \int_{0}^{1} I_{0}\left(r \xi \delta_{1}\right) \phi(r) \mathrm{d} r\right] \\
& \times\left\{2 \delta_{2}-n \exp (-m c \xi)-m \exp (-n c \xi)\right\} \int_{0}^{1} I_{0}\left(r \xi \delta_{2}\right) \phi(r) \mathrm{d} r \\
E_{2}(\xi) & \\
& \\
& \\
& \\
&
\end{aligned}
$$

where

$$
\begin{aligned}
p_{0}(\xi) & =n^{2}\{\exp (m c \xi)+\exp (-m c \xi)\} \\
& -m^{2}\{\exp (n c \xi)+\exp (-n c \xi)\}
\end{aligned}
$$

and

$$
n=\delta_{1}+\delta_{2}, m=\delta_{2}-\delta_{1} \text {. }
$$

Eliminating $D_{1}(\xi), D_{2}(\xi)$ and $E_{2}(\xi)$ from (36) we obtain the integral equation

$$
\phi(t)=\int_{0}^{1} K(t, r) \phi(r) \mathrm{d} r+G(t),
$$

where

$$
K(t, r)=t H(t, r)
$$

and

$$
\begin{aligned}
H(t, r)= & \frac{2 t}{m} \int_{0}^{\infty} \frac{\xi}{p_{0}(\xi)}\left\langle\left[\delta_{1} \delta_{2}\left\{\delta_{2} M_{0}\left(t \xi \delta_{2}\right)-\delta_{1} M_{0}\left(t \xi \delta_{1}\right)\right\}\right.\right. \\
& \times\{m(\exp (n c \xi)-\exp (-n c \xi))-n(\exp (-m c \xi)-\exp (m c \xi))\}
\end{aligned}
$$




$$
\begin{aligned}
& -\delta_{1} I_{0}\left(t \xi \delta_{1}\right)\left\{n \exp (m c \xi)-m \exp (-n c \xi)-2 \delta_{1}\right\}-\delta_{2} I_{0}\left(t \xi \delta_{2}\right) \\
& \left.\times\left\{n \exp (m c \xi)+m \exp (-n c \xi)-2 \delta_{2}\right\}\right]\left\{\delta_{2} M_{0}\left(r \xi \delta_{2}\right)\right. \\
& \left.-\delta_{1} M_{0}\left(r \xi \delta_{1}\right)\right\} \\
& -\delta_{2}^{2}\left[2 m \delta_{1}^{2} \exp (-n c \xi) I_{0}\left(t \xi \delta_{1}\right)\right. \\
& -m n \delta_{2} I_{0}\left(t \xi \delta_{2}\right) \exp (-n c \xi) \\
& -\exp (-m c \xi)+\delta_{1}\left\{\delta_{2} M_{0}\left(t \xi \delta_{2}\right)\right. \\
& \left.-\delta_{1} M_{0}\left(t \xi \delta_{1}\right)\right\}\left\{2 \delta_{2}-n \exp (-m c \xi)\right. \\
& -m \exp (-n c \xi)\}] I_{0}\left(r \xi \delta_{2}\right)+\delta_{1}^{2}\left[m n I_{0}\left(t \xi \delta_{1}\right)\{\exp (-n c \xi)\right. \\
& +\exp (m c \xi)\}+\delta_{2}^{2} I_{0}\left(t \xi \delta_{2}\right)\{m+n \exp (-m c \xi)\} \\
& \left.+\delta_{2}\left\{\delta_{2} M_{0}\left(t \xi \delta_{2}\right)-\delta_{1} M_{0}\left(t \xi \delta_{1}\right)\right\}\right] I_{0}\left(r \xi \delta_{1}\right)>\mathrm{d} \xi
\end{aligned}
$$

and

$$
\begin{aligned}
G(t) & =(2 / \pi)^{1 / 2} \frac{\delta_{2}}{A_{66}\left(1+\lambda_{2}\right)\left(\delta_{1}-\delta_{2}\right)} \frac{\mathrm{d}}{\mathrm{d} t} \int_{0}^{t} \frac{x \mathrm{~d} x}{\left(t^{2}-x^{2}\right)^{1 / 2}} \int_{0}^{x} \sigma^{0}(b, c ; v) \mathrm{d} v \\
& =(2 / \pi)^{1 / 2} \frac{\delta_{2} t}{A_{66}\left(1+\lambda_{2}\right)\left(\delta_{1}-\delta_{2}\right)} \int_{0}^{t} \frac{\sigma^{0}(b, c ; v) \mathrm{d} v}{\left(t^{2}-v^{2}\right)^{1 / 2}}
\end{aligned}
$$

Substituting $\sigma^{0}(b, c ; v)$ from (22) in (44) and using the formulae

$$
\frac{2}{\pi} \int_{0}^{t} \frac{\exp \left(-x \xi \delta_{i}\right)}{\left(t^{2}-x^{2}\right)^{1 / 2}} \mathrm{~d} x=I_{0}\left(t \xi \delta_{i}\right)-L_{0}\left(t \xi \delta_{i}\right)
$$

and

$$
\frac{2}{\pi} \int_{0}^{t} \frac{\exp \left(x \xi \delta_{i}\right)}{\left(t^{2}-x^{2}\right)^{1 / 2}} \mathrm{~d} x=I_{0}\left(t \xi \delta_{i}\right)+L_{0}\left(t \xi \delta_{i}\right)=R_{0}\left(t \xi \delta_{i}\right),
$$

we obtain

$$
G(t)=-\left(\frac{2}{\pi}\right)^{-1 / 2} \frac{P \delta_{1} \delta_{2} t^{2}}{\left(1+\lambda_{2}\right)\left(\delta_{1}-\delta_{2}\right)} \int_{0}^{\infty} g(\xi) \mathrm{d} \xi,
$$

where

$$
\begin{aligned}
g(\xi)= & \left\langle\delta _ { 1 } \left[\left\{\cos (b \xi)\left(X-Y \exp \left(-c \xi \delta_{2}\right)\right)+Y-X \exp \left(-c \xi \delta_{2}\right)\right\}\right.\right. \\
& \times M_{0}\left(t \xi \delta_{1}\right)+\left\{\cos (b \xi)\left(Y-X \exp \left(-c \xi \delta_{2}\right)+X-Y \exp \left(-c \xi \delta_{2}\right)\right\}\right. \\
& \left.\times \exp \left(-c \xi \delta_{1}\right) R_{0}\left(t \xi \delta_{1}\right)\right]+\delta_{2}\left[\left\{\cos (b \xi)\left(Y \exp \left(-c \xi \delta_{1}\right)-X\right)\right.\right. \\
& \left.+X \exp \left(-c \xi \delta_{1}\right)-Y\right\} M_{0}\left(t \xi \delta_{2}\right)+\left\{\cos (b \xi)\left(X \exp \left(-c \xi \delta_{1}\right)-Y\right)\right. \\
& \left.\left.+Y \exp \left(-c \xi \delta_{1}\right)-X\right\} \exp \left(-c \xi \delta_{2}\right) R_{0}\left(t \xi \delta_{2}\right)\right]>\Delta(c \xi)
\end{aligned}
$$

The stress intensity factor $K$ under three-point loading is defined as

$$
\begin{aligned}
K & =\lim _{x \rightarrow 1^{+}}(2(x-1))^{1 / 2}\left[\sigma_{y y}^{1}(x, 0)+\sigma_{y y}^{0}(x, 0)\right] \\
& =\lim _{x \rightarrow 1^{+}}(2(x-1))^{1 / 2} \sigma_{y y}^{1}(x, 0) \\
& =(2 / \pi)^{1 / 2} \frac{\left(1+\lambda_{2}\right)\left(\delta_{1}-\delta_{2}\right) A_{66}}{\delta_{2}} \phi(1) .
\end{aligned}
$$


Substituting

$$
\begin{aligned}
& \phi(t)=\left(\frac{2}{\pi} t\right)^{1 / 2} P \Psi(t), \quad 0 \leqslant t<1 \text { and } \\
& G(t)=\left(\frac{2}{\pi} t\right)^{1 / 2} P f(t), \quad 0 \leqslant t<1
\end{aligned}
$$

in (42) we obtain the simplified form of the same equation as

$$
(t)=\int_{0}^{1} M^{*}(t, r) \Psi(r) \mathrm{d} r+f(t),
$$

where

$$
M^{*}(t, r)=(t r)^{1 / 2} H(t, r)
$$

and

$$
f(t)=-\frac{\delta_{1} \delta_{2}^{2} t^{1 / 2}}{2\left(1+\lambda_{2}\right)\left(\delta_{1}-\delta_{2}\right)} \int_{0}^{\infty} g(\xi) \mathrm{d} \xi .
$$

Hence the stress intensity factor $K$ can be rewritten as

$$
\frac{K}{P A_{66}}=\frac{2}{\pi} \frac{\left(1+\lambda_{2}\right)\left(\delta_{1}-\delta_{2}\right)}{\delta_{2}} \Psi(1) .
$$

\section{Numerical results and conclusion}

Numerical study has been carried out in a high speed computer IBM 370 and good accuracy has been maintained. The integral equation (48) has been solved by the method developed by Fox \& Goodwin (1953). First the integral equation has been converted into a $10 \times 10$ system of algebraic linear equations. The first integral in (48) has been evaluated by Gauss-Laguerre quadrature formula while the second integral $f(t)$ has been numerically evaluated by Simpson's rule for integration and the procedure adopted by Sneddon (1951) for calculating the asymptotic value of the integral for large values of $\xi$, the variable of integration. While numerically evaluating the integral equation (48) for determining the value of stress intensity factor $K$, we have assumed

Table 1. Variation of stress intensity factors for isotropic and orthotropic materials for different values of $c$.

\begin{tabular}{lccc}
\hline$c$ & $\left(A_{66} P\right)^{-1} K$ & $(G P)^{-1} K$ & $(G P)^{-1} K$ \\
\hline 1.5 & 10.102 & 8.659 & 8.711 \\
1.75 & 6.505 & 5.576 & 5.610 \\
2.0 & 4.829 & 4.14 & 4.165 \\
2.5 & 3.245 & 2.779 & 2.796 \\
3.0 & 2.484 & 2.129 & 2.142 \\
4.0 & 1.737 & 1.489 & 1.498 \\
5.0 & 1.357 & 1.163 & 1.171 \\
\hline
\end{tabular}


$b=2 c$ and have used the value of material constants for boron-epoxy composite from Satapathy \& Parhi (1978). As theoretical derivation of the isotropic result from the orthotropic one is not possible, we have determined the value of stress intensity factor for the limiting case of isotropy, taking $A_{66}=0.99997$ which is 0.003 less than the actual isotropic value.

Table 1 shows the variations of the values of stress intensity factor for orthotropic, limiting isotropic and isotropic materials for different values of $c=1 \cdot 5,1 \cdot 75,2 \cdot 0,2 \cdot 5$, 3.0, 4.0 and 5.0. They are denoted as $\left(A_{66} P\right)^{-1} K,(G P)^{-1} K$ and $(G P)_{E}^{-1} K$ respectively.

Table 2. Elastic constants $A_{i j}$ for boron-epoxy composite

\begin{tabular}{cccccccc}
\hline$\frac{A_{11}}{10^{6} \mathrm{psi}^{*}}$ & $\frac{A_{12}}{10^{6} \mathrm{psi}}$ & $\frac{A_{66}}{10^{6} \mathrm{psi}}$ & $\frac{A_{22}}{10^{6} \mathrm{psi}}$ & $\lambda_{1}$ & $\lambda_{2}$ & $\delta_{1}^{2}$ & $\delta_{2}^{2}$ \\
\hline 30.3 & 3.78 & 1.13 & 4.04 & 17.430 & 0.057 & 2.862 & 0.047 \\
\hline
\end{tabular}

The direction of fibre is along the $x$-axis and $\lambda_{i}, \delta_{i}^{2}(i=1,2) ; 1 \mathrm{psi}=6.895 \times 10^{3} \mathrm{~Pa}$

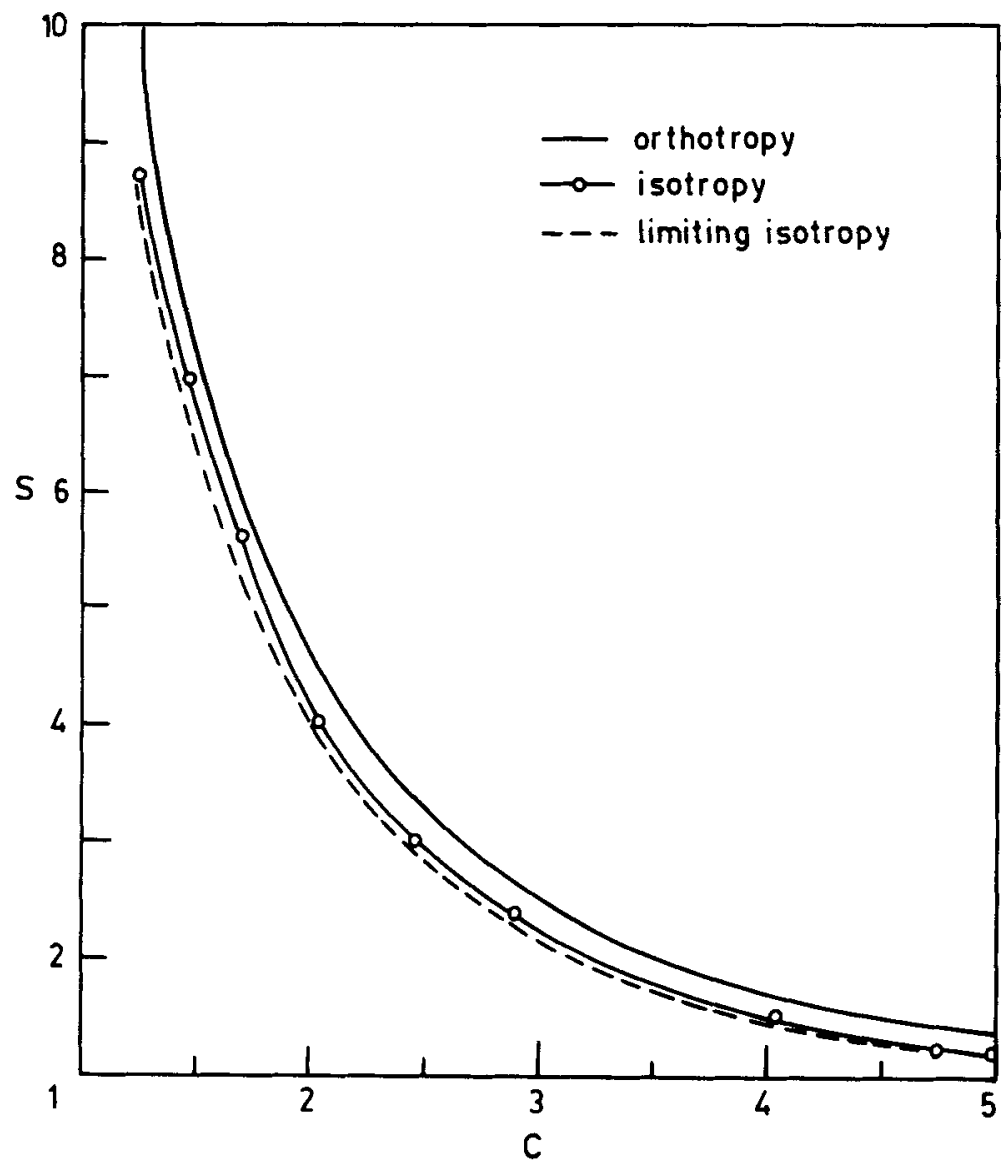

Figure 2. Variation of stress intensity factor with $\mathrm{C}$ 
It is evident from table 1 that the values of $(G P)_{E}^{-1} K$ for the isotropic case and $\left(A_{66} P\right)^{-1} K$ for the orthotropic case increase with the increase of $1 / c$ where $1 / c$ is the ratio of the crack length to the width of the strip. It is found that the rate of increase of values of stress intensity factor maintains uniformity for both the materials. Such increase becomes more rapid for $c<2.0$ or $1 / c>0.5$. So the strip becomes more sensitive to load as the width of the strip becomes less than twice the length of the crack. Further, the orthotropic strip is found to be more sensitive to load than the isotropic one. The value of $(G P)_{E}^{-1} K$, supplied in the table is the result taken from Ejike (1977). The result obtained for limiting isotropic material is found to be close to the value of isotropic one.

Table 2 shows the values of material constants for the boron-epoxy composite which we have utilised in the course of numerical evaluation. The values of stress intensity factor for orthotropy, isotropy and limiting isotropy have been tabulated in table 1 and shown as graphs in figure 2 .

\section{References}

Bowie O L 1964 J. Appl. Mech. 31: 208-212

Delate F, Erdogan F 1977 J. Appl. Mech. 44: 237-243

Ejike U B C O 1973 Int. J. Eng. Sci. 11: 109-120

Ejike U B C O 1977 Int. J. Eng. Sci. 17: 413-420

Fox L, Goodwin E T 1953 Philos. Trans. R. Soc. A245: 501-533

Gupta G D, Erdogan F 1974 J. Appl. Mech. 41: 1001-1006

Koiter W T 1965 J. Appl. Mech. 32: 257-263

Kudryavtsev B A, Parton V Z 1973 Prikl. Mat. Mek. 37: 316-325

Satapathy P K, Parhi H 1978 Int. J. Eng. Sci. 16: 147-154

Sneddon I N 1951 Fourier transforms (New York: McGraw-Hill) 